\title{
La relevancia de auditar requisi- tos de información en el diseño de sistemas de gestión de docu- mentos. Métodos tradicionales, enfoques emergentes
}

\author{
María M. Moro Cabero *
}

Artículo recibido:

19 de noviembre de 2010.

Artículo aceptado:

6 de abril de 2011.

\section{RESUMEN}

El presente ensayo justifica el análisis de requisitos de información frente a un contexto de cambio en las organizaciones y enumera los factores que inciden en su evolución. Se investigan y describen los principales métodos y técnicas empleados en la auditoría de requisitos, señalando los enfoques emergentes más destacados. También se enuncian algunas experiencias internacionales y nacionales y se incorporan sugerencias de trabajo. Para la elaboración de este texto se han revisado y analizado recursos bibliográficos específicos así como normativos: series ISO de gestión de calidad, de seguridad, de riesgos y de gestión documental. El método empleado es analítico-descriptivo.

* Universidad de Salamanca, España. moroca@usal.es

INVESTIGACIÓN BIBLIOTECOLÓGICA, Vol. 25, Núm. 53, enero/abril, 2011, México, ISSN: 0187-358X. pp. 201-230 
Palabras clave: Auditoría de requisitos de gestión documental; Gestión de documentos; Normativa ISO 15489:2001; Métodos y técnicas de auditoría.

\section{ABStract}

The importance of audit information requirements in the design of records management systems: Traditional methodologies and emerging approaches María M. Moro Cabero

This essay provides a rationale for the analysis of information requirements in a context of organizational change and gives an account of the factors that influence its evolution. The prevailing, most relevant approaches, methods and techniques for auditing records requirements are described, along with several local and foreign cases with suggestions for future developments. Employing an analytical-descriptive approach, this paper is underpinned by a review and analysis of specialized bibliography and ISO standards on security, risks, and records auditing and management.

Key Words: Records system auditing; Records Management; Standard ISO 15489:2001; Methodologies and techniques of auditing.

\section{INTRODUCCIÓN}

E 1 borrador ISO/DIS 30301:2010 de revisión de la normativa ISO 15489:2001, ${ }^{1}$ sobre gestión de documentos, incide en la necesidad de planificar, mantener e implementar un sistema de gestión de documentos que permita la continuidad de los negocios de la organización que cumplan con las políticas estatuidas y demuestren conformidad con otros sistemas de gestión activos en la organización. ${ }^{2}$ El método utilizado se sustenta sobre el conocido ciclo de gestión PDCA: planificación, diseño, control y actuación hacia la mejora,

1 Las normas consultadas de la serie ISO 15489:2001 se corresponden con la traducción realizada por AENOR (Asociación Española de Normalización) -código UNE-.

2 ISO TC46-SC11-WG9: ISO/ DIS 30301, Management system for records-Requirements; 2010. Apartado 1, p. 1. 
promovido por W. Shewart y mejorado por E. Deming. ${ }^{3}$ Ciclo de gestión adoptado igualmente en los principales códigos de gestión de sistemas de calidad (serie ISO 9000 4 ), de sostenibilidad medioambiental (serie ISO 14000) y de seguridad de la información (serie ISO 27000).

El estudio se centra en la fase de planificación con una doble finalidad: verificar la importancia de la medición en el proceso planificador para identificar requisitos de gestión documental, y describir los métodos y técnicas empleados en la auditoría de sistemas de gestión documental, enfatizando aquellos enfoques emergentes en los métodos y técnicas empleados. Su finalidad es aportar una reflexión retrospectiva y prospectiva sobre dicho proceso.

Entendemos por auditoría (informativa) un examen profundo, metódico y planificado de un sistema, una unidad, un proceso, un servicio o un producto (informativo). En esta línea, C. Soy (Soy Aumatell; 2003, p.35) entiende que la auditoría informativa "establece las necesidades de información de la organización, determina cómo dan respuesta los recursos de información a estas necesidades y establece unas pautas de mejora del recurso información”. La normativa ISO 19011(2002; p. 9) aporta un concepto que se corresponde más con la fase de control del ciclo PDCA que con la de planificación, al definirla como:

Proceso sistemático, independiente y documentado para obtener evidencias de la auditoría y evaluarlas de manera objetiva con el fin de determinar la extensión en que se cumplen los criterios de auditoría.

Una lectura atenta de la serie ISO 9000 nos permite comprender que el concepto puede ser utilizado en ambas fases en función de su finalidad: análisis de los requisitos y de su cumplimiento (planificación), y análisis de la conformidad y de su rendimiento (evaluación).

La planificación se sustenta sobre una secuencia que enumeramos seguidamente: a) formulación de objetivos de planificación delimitando alcance y concreción sobre aquello que será planificado; b) recopilación de datos para conocer los contextos; c) reflexión sobre la información obtenida para elaborar un diagnóstico d) elaboración de pronósticos y coordinación de recursos; e) establecimiento de un diseño preliminar basado en estrategias de diseño, implantación y control. Esta última fase nos predispone para

3 Ibidem, p. VII.

4 Con similar expresión, aunque para un sistema de gestión de calidad, es posible observar en el apartado 4.1 de la norma ISO 9001:2008. "la organización debe establecer, documentar, implementar y mantener un sistema de gestión de calidad y mejorar continuamente su eficacia..." (p.12). 
acometer el diseño, dándole continuidad de este modo a la secuencia de fases del ciclo PDCA.

El trabajo subraya la importancia de la auditoría en el diseño de sistemas de gestión de documentos y reflexiona sobre los métodos y técnicas que se desvelan en la normativa ISO 15489:2001, así como en su desarrollo normativo complementario: UNE-ISO/TR 26122:2008, para proceder a la planificación. El ensayo enuncia aquellas técnicas que desde "el corazón de la organización" son percibidas en la actualidad como factores activos de progreso para la propia organización. Su análisis se ha fundamentado sobre la bibliografía de referencia en el sector, así como sobre la serie ISO 15489:2001 y sus más recientes borradores, producto de su revisión.

Para presentar dicha investigación se ha optado por una estructura que se inicia justificando la importancia de la actividad auditora en el contexto profesional. En segundo término se reflexiona sobre el conjunto de requisitos informativos en un contexto de incertidumbre: requisitos que aportan nueva asimetrías y contribuyen a aumentar los significados en la actividad de archivar, y que hemos caracterizado con el término "factores deslocalizadores". El objetivo es identificar y analizar de su alcance e impacto en el aseguramiento de un archivo sostenible. ${ }^{5}$ En tercer lugar, se exponen los principales métodos y técnicas asociadas de auditoría, indicando las novedades extractadas de las propuestas normativas. Mediante este enunciado pretendemos dar a conocer métodos y técnicas, a la par que aportamos una reflexión sobre aquellas técnicas que son reinterpretadas o utilizadas con diferentes fines a los tradicionalmente buscados. Un cuarto epígrafe enuncia algunas de las experiencias internacionales y nacionales con el fin de procurarle al lector interesado en el ejercicio auditor, un referente práctico y real de trabajo. Finalmente, se aportan algunas conclusiones y propuestas abiertas de investigación.

\section{JUSTIFICACIÓN Y MOTIVACIÓN DE UNA ACTUACIÓN AUDITORA: el archivero en la encrucijada ante el Gato de Cheshire. ${ }^{6}$}

La adopción por una organización de la serie ISO 15489, específica sobre administración de sistemas de gestión documental, se promueve con base en los efectos que se pretenden alcanzar con su implementación. Los beneficios

5 Retomamos para este calificativo de "sostenible" el concepto medioambiental aplicado al archivo: el archivo debe satisfacer las necesidades de las generaciones actuales sin privar a las generaciones futuras de la posibilidad de satisfacer sus propias necesidades.

6 Figura enigmática que aparece en el libro de C. Lewis. Véase Carroll, Lewis: Alicia en el País de las Maravillas, Ilustrado por H. Oxenbury, Barcelona: Círculo de lectores, 1999. 
consignados en la norma ISO 15489-1 (2001:p 5 y s.) se orientan a: facilitar el desarrollo de las actividades del negocio y prestar servicios de modo eficaz y responsable; proteger intereses de la organización mediante el apoyo en los litigios y el cumplimiento con los requisitos administrativos, legales y otros derivados de códigos y disposiciones; identificar y gestionar posibles riesgos mediante la adopción de programas activos de gestión de documentos esenciales y de gestión de desastres; respaldar y documentar directrices y toma de decisiones; proporcionar evidencias, documentar las actividades y procesos de gestión, y mantener una memoria patrimonial sobre la corporación u otros sujetos y eventos de interés para la sociedad.

Del conjunto de beneficios derivados de la implementación de un sistema de gestión de documentos destacamos, en nuestra opinión, el aseguramiento de la transparencia y trazabilidad sobre las decisiones adoptadas, el mantenimiento de la continuidad de las actividades realizadas, y también la contribución al cumplimiento de los requisitos documentales administrativos, legales y derivados de diversos códigos adoptados de buenas prácticas de gestión (calidad, seguridad, sostenibilidad medioambiental) y de otros códigos éticos (transparencia, buen gobierno, responsabilidad social). Para lograr esto, la propuesta normalizada incluye una serie de estrategias de las cuáles enfatizamos: el alineamiento de la gestión de los documentos con los negocios de la organización; el establecimiento de un sistema de distribución de responsabilidades y rendición de cuentas; el alineamiento de la gestión documental con otras áreas de evidente interés: calidad, seguridad, tecnologías de la información, auditoras, etc.; el conocimiento y control de los entornos — externos e internos — administrativos y legales en los que opera la organización con el fin de identificar requisitos de cumplimiento y, finalmente, en una aproximación sistémica de la gestión con la regulación conceptual y metodológica de un sistema de gestión documental. La implementación y mantenimiento de un sistema de estas características exige una rigurosa definición y asignación de funciones y de responsabilidades, el despliegue de una arquitectura de procesos, el control y medición de los logros, y una revisión orientada a la mejora continuada.

La serie ISO 15489: $2001^{7}$ aporta un método —normalizado- de administración del sistema de gestión documental. En este hecho radica su innovación. Su aplicación repercute en el enfoque dado al ejercicio de "archivar", multiplicando la perspectiva; esto es, los modos de comprender qué, quién, para qué, por qué, dónde, cómo y con qué se han de gestionar los documentos en

7 En este estudio se hace constante referencia a la normativa editada, aunque siempre que se considere pertinente por su carácter novedoso se hará referencia a los últimos borradores de revisión de la serie. Considérese al respecto que dichos borradores se encuentran en una fase de elaboración muy avanzada (Formato DIS- abierto a discusión internacional). 
una organización, durante su vida activa y en el Archivo, ${ }^{8}$ una vez considerados de valor permanente. Las estrategias propuestas por la antedicha normativa ISO se justifican al retomar el neologismo, creado por E. Katelaar (2001:p.132), de "archivalizar", definido por dicho autor como "la elección consciente e inconsciente 9 para considerar si algo merece ser archivado". De este modo, mediante la distinción del término archivar — gestionar documentos - del vocablo archivalizar, -interacción de múltiples significados en el ejercicio de gestionar los documentos-, Katelaar nos induce a reflexionar sobre la diversidad de asimetrías que es posible observar en un Archivo (y, por ende, en un sistema de gestión de documentos activos en una organización). ${ }^{10}$ En suma, constatamos, utilizando el término de Katelaar para tal fin, que las estrategias anteriormente citadas son producto de rechazar una verdad única, una realidad única, un significado único. A este respecto, la investigación de la genealogía semántica de un archivo nos conduce a estudiar el contexto interno y externo — administrativo, jurídico, económico, social, político y cultural - de una organización inserta en una sociedad, con el objetivo de comprender la formación de las antedichas asimetrías y de reconocer el camino que el archivero debe recorrer en su ejercicio “archivalizador”. Un recorrido que Llansó Sanjuán (2009) recomienda transitar, utilizando como metáfora al archivero-Ulises en su largo periplo por los mares (de la organización) antes de regresar a Ítaca (el Archivo). Llansó utiliza los bellos versos de C.P. Cavafis para recordar que el navío debe de estar abanderado por la normalización, invitando al archivero a navegar por ignotos mares y a regresar "convertido en tan sabio, y con tanta experiencia" que le permita comprender "el significado de las Ítacas". ${ }^{11}$

La normativa propone, dado el contexto cambiante en el que interactúan las organizaciones, incipientes modos de administrar un sistema de gestión documental. Se constituye en prototipo de administración distribuida en la organización así como de asignación y delegación de las responsabilidades sobre la gestión documental, para cuya concreción se requiere aplicar de modo novedoso métodos y técnicas de administración. A este respecto, el gestor de documentos, entretenido en su tradicional modo de archivar, deberá asumir

8 Retomamos la diferencia observada por A. Heredia entre Archivo y archivo (Heredia Herrera; 2007:p.19 y ss.) destinando el concepto Archivo, con mayúscula, para la institución que se crea y administra - unidad informativa- por una institución. El uso del término archivo, con minúscula, se destina para el conjunto de documentos que deben ser gestionados y son producidos por una organización.

9 Determinada por todo tipo de factores: sociales, culturales, políticas, etcétera.

10 El archivo entendido en sus múltiples acepciones como un conjunto de documentos activos y operativos en una organización o como unidad de información de una organización donde pueden conservarse documentos activos, semiactivos o inactivos con valor de conservación permanente.

11 Cavafis, C.P. Poemas, Traducción y prólogo de Ramón Irigoyen, Barcelona: Círculo de Lectores, 1999, Poema Ítaca, p. 66. 
una desconocida encrucijada y, con idéntico proceder al experimentado por Alicia en el País de las maravillas, habrá de enfrentarse al dilema de seleccionar un camino que le permita responder a los estrenados requisitos de "archivar" presentados por las organizaciones, comprendiendo sus nacientes significados; esto es: deberá archivalizar, empleando el neologismo de Katelaar. La cuestión esencial radica en dilucidar cómo empleará la respuesta ofrecida por el gato de Cheshire en su profesión de gestor de documentos. Recordemos que el animal de enigmática sonrisa propone una velada contestación al interrogante planteado por Alicia: "Esto depende del sitio al que quieras llegar" ${ }^{2}$ ¿A dónde pretenden llegar los gestores de documentos? ¿De qué bosque prefieren salir? ¿Qué mares están dispuestos a surcar?

A. D’Alós (2001: p26), al reflexionar sobre la profesión en el siglo XXI, destaca que: "ya no es suficiente gestionar de manera más o menos eficaz un servicio de información, sino que hay que ser capaz de situarse en el corazón de la organización, ser un elemento central que incide en su crecimiento". Similar opinión nos brinda L. Esteve Casellas (2009:p45) cuando señala que:

la reivindicada visibilidad social de la profesión se obtendrá más por las funciones que desempeñemos en nuestras organizaciones que por nuestra labor en los archivos históricos, pero sin olvidar nuestra función cultural.

Este modo de entender la actuación del profesional ante la información en las organizaciones, caracteriza a un gestor que pretende llegar a un prototipo de servicio integrador y corporativo, a la par que presupone lograr el diseño de un modelo de gestión de documentos "sostenible"; esto es, armónico: que sea capaz de disponer la información y de contribuir al mantenimiento de una dinámica de relaciones favorables entre la organización y su entorno en continua interdependencia. ¿Qué consecuencias se infieren ante el abandono del viejo bosque? El logro de una gestión sostenible de los documentos en una organización requiere investigar las transformaciones originarias y constantes del entorno interno (organización) y externo (realidad jurídico-política y económico-social); dinámicas y alteraciones que repercuten directamente sobre su sistema de gestión documental. ${ }^{13}$ Este proceso investigador se concreta en una auditoría de requisitos de información, cuya finalidad es la formulación de objetivos y la identificación de estrategias para (re) diseñar, implementar y evaluar un sistema de gestión documental. Auditar requisitos y formular objetivos de gestión de la información caracterizan

12 Opus cit. p.102

13 El modelo normativo ISO/DIS 30301 así lo refleja, incluyendo un apartado específico destinado al contexto de la organización. Véase apartado 4, p. 2 y ss. 
la planificación de un sistema de gestión documental: primera fase imprescindible del ciclo de gestión PDCA.

El método de gestión, basado en el ciclo PDCA, propuesto en la normativa, debe considerarse novedoso para el profesional por el mero hecho de fundamentarse de modo normalizado y consensuado a nivel internacional en una comunidad profesional, donde la especificidad de modelos de gestión es considerada un requisito en la construcción de cualquiera de ellos.; si bien, el dilema fundamental es conocer hasta qué punto, los archiveros han empleado y dominan sus técnicas; se trataría de, averiguar el grado de conocimiento que poseen de las técnicas de desbroce del bosque, o de orientación marítima hacia Ítaca; en suma, conocer su grado competencial para ubicarse en el corazón de la organización. Los archiveros, capaces de sobrevivir a tres milenios de práctica preservadora de documentos, han sabido identificar y coordinar durante milenios estrategias (acciones planificadoras) y diseños (respuestas creativas). Buena parte de las técnicas empleadas en algunas de las fases del ciclo PDCA son técnicas conocidas por los administradores de sistemas de archivo. ¿Qué tipo de innovación nos invita actualmente a reflexionar sobre esta fase del ciclo de gestión? En la práctica tradicional de la gestión documental, la acción auditora ha estado presente en la comunidad archivística, tal y como es posible observar en los principales manuales de gestión. ${ }^{14}$ En ellos, se detallan técnicas específicas orientadas a facilitar

14 La metodología en la práctica de gestión documental angloamericana se halla con mayor o menor alcance detallada en buena parte de sus clásicos manuales, de los cuáles referenciamos:

Para Estados Unidos, se proporcionan dos referencias en las que se disponen en cada una de ellas de 3 capítulos. Estas son: a) Ira A. Penn, Ira A., Pennix, Gail B. y Coulson, Jim. "Records Management Handbook", Vermont: Gower; 1994. b) Active Filing for Business Records, Ann Bennick, ed. Prairie Village: ARMA; 2000.

En la práctica canadiense se incorpora información tanto en los manuales de C. Couture $\left(1^{\mathrm{a}}\right.$ y $2^{\mathrm{a}}$ ed.) -agrega un capítulo específico, como en el manual de M. Robérge. Se hace referencia a la $2^{a}$ ed. De Couture, C- y colaboradores: Les fonctions de l'archivistique contemporice, Québec, Universidad de Québec; 1999. De las diferentes ediciones del manual de M. Robérge, se detalla mediante un capítulo específico el inventario físico de los documentos, únicamente, en la primera monografía de 1983. Se omite dicho capítulo tanto en la $2^{\mathrm{a}}$ (1992) como en la $3^{\mathrm{a}}$ (2002) edición de este manual. Para el lector interesado se referencia la primera edición del autor: Roberge, M., La gestión de l'information administrative: application globale, systémique et systematique, Quebec: Documentor, 1983.

En Australia, mientras que el manual de J. Ellis, editado en 1993 no incluye metodología sobre auditoría, el manual DIRKs de los Archivos Nacionales de Australia, editado en el 2001, especifica, mediante 3 capítulos y 10 apéndices, el proceso de auditoría. Véanse las siguientes obras: Keeping Archives, ed. Judith Ellis, $2^{a}$ ed, Melbourne (Australia), Thorpe, 1993, y Archivos Nacionales de Australia: Designing and Implementing Recordkeeping Systems (DIRKS) Manual; (en línea) [http://www.naa.gov.au/records-management/publications/DIRKS-manual. aspx ].

De igual modo se citan monografías específicas: La Fundación Internacional de Gestión de Documentos editó en 1999 la siguiente referencia: International Records Management Trust: Analising Business Systems, Ed. IRMT; CIA. (en línea) [http://www.irmt.org]. 
el inventariado de documentos, así como la identificación de los contextos de producción, desde la perspectiva más tradicional del concepto de gestión documental: búsqueda de rentabilidad económica en el almacenamiento y recuperación del documento. Si bien cabe señalar que en general la perspectiva social del patrimonio documental estaba ausente y prevalecía, únicamente, la visión centrada en el documento propiamente dicho, supeditando sus límites a las necesidades informativas departamentales y no tanto a su función corporativa y social.

Los recientes contextos productivos han conducido al gestor de documentos hacia el corazón de la organización, deslocalizando el archivo, ${ }^{15}$ hacia la exterioridad, hacia su afuera (retomando la idea de Derrida ${ }^{16}$ ). Le han permitido abandonar el bosque ubicándose en una vereda distinta en la que se vislumbra otro paisaje, dado que, junto al factor tangible de la rentabilidad económica, ${ }^{17}$ es posible observar la gestión de la información como activo intangible en la organización. Una amplia correlación de costos y beneficios cualitativos ensanchan el paisaje, modificando la actividad del paisanaje. El propósito no se centra en la rentabilidad económica del objeto documento, sino en la administración del sistema de gestión documental, al que ante todo, se le ha asignado una misión estratégica de servicio continuado y permanente en la organización, sin descuidar su contribución a la preservación del patrimonio. Este hecho ha incidido en el desarrollo de nuevas reflexiones ante los cambios y en la elaboración de estándares, cuya finalidad es regular la fase mediante la provisión de métodos y de técnicas normalizadas de trabajo para ejercer los procesos auditores. La relación de factores que han contribuido al cambio se analiza en el epígrafe siguiente.

\section{Factores deslocalizadores en un Contexto de Cambio}

EN LAS ORGANIZACIONES: EL ARCHIVERO AFRONTANDO INCERTIDUMBRES INFORMATIVAS

Damos inicio a este epígrafe mediante la referencia al pasaje utilizado por L. Carroll en el que Alicia conversa con la oruga sobre cómo controlar su (de) crecimiento:

15 La deslocalización debe entenderse como el cambio de lugar del centro de funcionamiento y de producción motivado por la búsqueda de mayor rentabilidad sobre los recursos materiales, humanos, etc. y, por lo tanto, sobre los resultados económicos o de inversión.

16 Derrida, Jacques: Mal de archivo. Una impresión freudiana, Conferencias pronunciadas en1994, Edición digital de Derrida en Castellano, Editado en Londres, 1996, [accesible en línea].

17 Agilidad en la recuperación y rentabilidad de espacios y soportes. 
- Un lado te hará crecer y el otro disminuir

- Un lado ¿de qué? Y el otro ¿de qué? Se dijo Alicia para sus adentros. ${ }^{18}$

Al igual que Alicia debe enfrentarse a la circunferencia de una seta, el archivero, en su ejercicio profesional, debe afrontar los ciclos y contextos de cambio que experimentan las organizaciones. Alicia alarga sus brazos circunvalando el hongo con objeto de averiguar sus efectos bipolares y, de este modo, poder controlarlos y disfrutarlos. El archivero ante un contexto de cambio organizacional ha de investigar la evolución de los requisitos informativos de una organización. El concepto de requisito empleado en los entornos de gestión de calidad, y entendido como necesidad o expectativa establecida, generalmente implícita u obligatoria (UNE-ISO 9000:2005: apartado 3.1.2) es aplicado en el contexto de la información para definir el conjunto de necesidades informativas o expectativas establecidas — de modo implícito o explícito- sobre el aseguramiento de la evidencia documental, la disponibilidad, y la preservación de documentos, así como sobre la prestación de servicios de información asociados a una unidad de información o a un sistema de gestión documental.

El conocimiento de los requisitos de gestión documental en una organización se percibe como un factor estratégico ante el diseño de un sistema de gestión documental rentable y orientado al logro de dichos requisitos, - o de aquellos cuyo cumplimiento es considerado imprescindible-, con objeto de alcanzar, mantener y mejorar un rendimiento satisfactorio, tanto para los usuarios como para la organización, en general.

Estos requisitos de gestión documental son cambiantes y heterogéneos en atención a la diversidad de organizaciones, a la confluencia de múltiples factores asociados a la información, y a la pervivencia de diferenciados niveles de su tratamiento, disposición y conservación. De este modo, la información adquiere gran importancia al considerar aspectos tales como cantidad, calidad, seguridad, sostenibilidad, transparencia, ética, responsabilidad social, buen gobierno o, incluso, al estimar la naturaleza misma de las realidades específicas en las que se imbrican las organizaciones productoras. En nuestra sociedad Red, el volumen de información generada y utilizada durante la toma de decisiones es cada vez mayor y adquiriere vital transcendencia en la administración de negocios. La ineficacia y la lentitud en las decisiones son penalizadas. Los procesos de control, actualización y servicio de los documentos exógenos y endógenos conllevan asociados tanto determinados costos adicionales, como beneficios cuantificables, de carácter cuantitativo y 
cualitativo. A pesar de ello, nos recuerda C. Bustelo (2003: p. 119), son cuantiosas las organizaciones que no han sabido adaptar los modos de organizar, conservar y servir la información a su exponencial crecimiento y a las nuevas demandas de almacenamiento y recuperación; razón por la que las viejas fórmulas utilizadas para gestionar la información no les funcionan en el presente; hecho que las obliga a orientarse hacia la auditoría de la calidad de sus métodos y hacia la búsqueda de soluciones de mejora.

Esta tendencia de adaptación adquiere mayor relevancia si consideramos que en los entornos de trabajo empresarial inciden múltiples situaciones sobre las que una eficiente gestión de la información repercutirá de modo estratégico.

Un ejemplo característico es observable en el grado de obsolescencia de los ciclos de negocio - acortándose los plazos entre la idea y la comercialización del producto-servicio en semanas- debido a la competitividad de los negocios, para cuyo éxito y capacidad de respuesta se requiere una dinámica informativa basada en el flujo de datos; una eficiente circulación favorecerá intervenciones rápidas e inteligentes y permitirá la innovación. El fundamento del éxito de los negocios se encuentra en la información propia sobre clientes, proveedores, estrategias, ventas, contextos de mercados y competidores.

Un segundo ejemplo sobre la relevancia y dinamismo de los requisitos se percibe ante el surgimiento de requisitos informativos inéditos derivados de la adopción de códigos normalizados de gestión de sistemas (calidad, medioambiente, seguridad) y otros códigos de buenas prácticas (ejemplo: responsabilidad social y buen gobierno, transparencia, etc.), para cuya implantación y mantenimiento se requiere el cumplimiento de determinadas necesidades de gestión documental. Así, el modelo normalizado de gestión de sistemas de calidad dispone de un informe técnico específico para la regulación de la gestión documental del sistema (ISO 10013:2001). La importancia de la información ya se reflejaba en la serie ISO 9000:2000 cuando se articulaban las obligaciones de la dirección del modo siguiente:

La dirección debería tratar los datos como un recurso fundamental para su conversión en información y para el desarrollo continuo del conocimiento de una organización, el cual es esencial para la toma de decisiones basada en hechos y además puede estimular la innovación". ${ }^{19}$

En este orden, el borrador ISO/DIS 30300 (2010:p8) incluye un apartado específico sobre las relaciones con otros sistemas de gestión señalando las siguientes 
ventajas: "eliminación de redundancias, mejora en la toma de decisiones, contribución a una mayor consistencia del sistema documental, colaboración en el cumplimiento de los requisitos documentales y optimación de procesos y de recursos". 20

La relevancia de auditar los requisitos informativos se justifica, además, porque en las organizaciones concurren heterogéneos perfiles o modelos de gestión de la información; mientras que, algunas de estas organizaciones, todavía se ciñen al mero nivel operativo de manejo de datos, centrando su gestión en la tarea o conjunto de tareas asignadas a un puesto de trabajo y administrando puntualmente los problemas diarios, una gran mayoría de organizaciones, a nuestro juicio, han consolidado un modelo de gestión de datos, de medidas y de registros o estándares específicos reconocibles en la función o departamento: organizando dicha información en el ciclo de la función y/o ámbito departamental. Otras, en un número más reducido, sin embargo, tienden a integrar la información respetando una actuación corporativa y asumiendo otros requisitos informativos funcionales o necesidades afines a otros departamentos y/o procesos en su conjunto, para hacer factible ciclos anuales de administración corporativa. Un porcentaje muy menguado tiende a generar modelos de aprovechamiento optimado de la información en las corporaciones y el mercado exterior. En esta línea, J. Davis, G. Millar y A. Russell (2008: p28) enuncian que un 70\% de las organizaciones no sobrepasan el nivel informativo de actuación departamental. ${ }^{21}$

A la luz de estos datos, es posible confirmar cierta "exterioridad del Archivo" donde concurren un conjunto de necesidades y expectativas de información (y de su administración), originadas por el aumento exponencial de la información, aunque también por la importancia que la valoración científica de dicha información ha adquirido (uso, soporte, espacio de almacenamiento y/o preservación). Asimismo los requisitos se multiplican debido a la primacía de asegurar evidencias de las operaciones y transacciones de negocio vigentes y retrospectivas con objeto de subrayar el patrimonio institucional o la memoria social del territorio; los requisitos informativos, igualmente, son valorados dada la rapidez exigida en la recuperación de la información estratégica

20 Estudios específicos sobre la interrelación con la serie de calidad, véase: Moro Cabero, M., "El código (ISO 15489:2001) y el fuero (normativa ISO 9000): la importancia de gestionar documentos en entornos de gestión de calidad", en Homenaje a Isabel de Torres Ramírez: Estudios de Documentación dedicada a su memoria, Universidad de Granada. 2009, pp. 517-540. En el borrador ISO/DIS 30301, Anexo A, se incluye una tabla donde se muestra la interrelación con los requisitos documentales y otros sistemas de gestión normalizados en ISO. (9001, 14001, 27001).

21 Estos autores establecen una escala de 5 niveles atendiendo a su alcance y naturaleza (señalamos de menor a mayor: acción puntual, aunada a la tarea, acción departamental, nivel integrador corporativo, nivel de optimización de mercados y nivel innovador) ubicando a la mayoría de las organizaciones en el segundo nivel. 
y operativa con el fin de minimizar riesgos económicos por pérdidas de tiempo, disminución de la precisión, ajuste de información corporativa, pérdida de clientes o insatisfacción ante un servicio inadecuado. De igual modo, dicha exterioridad condiciona el cumplimiento de múltiples leyes y reglamentos locales, autonómicos, federales, nacionales e internacionales que regulan la creación, almacenamiento, manipulación, acceso y disposición de la información.

Por otro lado la gestión documental ha de moldearse asumiendo la concurrencia de heterogéneas realidades existentes en las organizaciones, dado que estas últimas son generadoras de información que actúa desde múltiples e interactivas dimensiones, entre las que destacamos por su grado de incidencia:

a) la infraestructura tecnológica y de comunicación, el canal de procesamiento, el almacenamiento, la difusión y la conservación de la información;

b) la cultura corporativa o culturas predominantes de la institución que caracterizan valores en torno a la transparencia, el buen gobierno, la calidad del servicio o la producción, la seguridad de la información, la disponibilidad de los datos, etcétera;

c) la concepción de la gestión del conocimiento organizacional, favorecedora de la adopción de políticas y del despliegue de una arquitectura de procesos, $y$

d) el capital humano, cuyas competencias y calidad de vida laboral deben ser auditadas en beneficio de una administración de la información distribuida, responsablemente asignada y/o delegada.

Precisamente serán los ajustes de estas dimensiones lo que perfilarán la multiplicidad de niveles de tratamiento, conservación y disposición de la información a la que anteriormente se ha aludido. La heterogeneidad de los contextos narrativos analizados en las organizaciones potencian la variedad de requisitos informativos a la par que multiplican los significantes percibidos por los gestores en la organización. De hecho en el manual australiano DIRKS se enumeran tres categorías de requisitos de gestión asociados al:

a) cumplimiento legislativo, reglamentario y normativo; entorno interno (acuerdos, órdenes, reglamentos y procedimientos) y externo a la organización (legislación, convenios, especificaciones, códigos de buenas prácticas y normativas diversas). Estas categorías han sido 
estudiadas y pautadas en monografías específicas (Skupsky; 1994) ${ }^{22}$ normas $^{23}$ y manuales; ${ }^{24}$

b) apoyo operativo a los procesos de negocio; entorno interno de la organización: organización del trabajo, y

c) logro de las expectativas de la comunidad (entorno interno y externo: productores, clientes y terceras partes).

En esta línea, el borrador ISO/DIS 30300 (2010:p.2) señala la operatividad de dicha norma para el reconocimiento de las expectativas y necesidades de cualquier tercera parte o interesado así como potenciales requisitos futuros de dichos interesados. Reincidiendo en esta idea, el borrador ISO/DIS 30301 (2010: p 3), incluye un enunciado específico, cuyo título resulta bastante esclarecedor: "Business, legal and other requirements", e incorpora en él las expectativas de la comunidad.

\section{MÉtodo Normalizado de AUditoría de REQUisitos: ETAPAS Y TÉCNICAS ASOCIADAS.}

\subsection{Presentación del método auditor}

La metodología desplegada en la parte 1 de la norma ISO 15489-1:2001, —específica a la planificación del sistema-, define el método de trabajo auditor del siguiente modo: recolección de datos; análisis de la información recopilada; identificación de los requisitos de información, y formulación de estrategias de gestión documental, con el fin de diseñar, implementar y evaluar un programa de gestión de documentos. Cuatro son por tanto las etapas del método:

a) recolección de datos del contexto organizacional sobre el programa de gestión de documentos y sobre las necesidades reales y potenciales de información,

b) análisis e interpretación de los hallazgos ${ }^{25}$ investigados;

22 Destina varios capítulos a señalar consecuencias ante el incumplimiento, y estrategias para lograr su cumplimiento.

23 El apartado 5 de la norma ISO 15489:2001, Parte 1, regula el entorno normativo. Igualmente, la norma UNE-ISO TR 26122:2008 referencia en su metodología las fuentes legislativas y normativas como base para investigar la organización del trabajo y asumir los diferentes tipos de análisis que regula.

24 Tanto el Manual DIRKS como el de la IRMT aluden a las fuentes legislativas.

25 Utilizamos el concepto aportado en la norma UNE-ISO 19011:2002, que lo define como: "Resultados de la evaluación de la evidencia de la auditoría recopilada frente a los criterios de auditoría”. p. 10. 
c) identificación de los requisitos de información y formulación de las estrategias para el diseño de un sistema de gestión de documentos;

d) comunicación de resultados mediante procedimiento verbal y/o documentado para la toma de decisiones sobre el diseño o mejora.

El código enuncia una serie de operaciones que seguidamente se señalan asociadas a las etapas del método auditor: ${ }^{26}$

a) investigación preliminar (etapa de recolección de datos);

b) análisis de las actividades de la organización y evaluación de los sistemas de archivo (etapa de análisis e interpretación de datos);

c) identificación de requisitos de los documentos, identificación de estrategias y evaluación de los sistemas de archivo (etapa de identificación de requisitos y de formulación de estrategias), y

d) a su vez, en la normativa se insiste -de modo persistente- en la necesidad de documentar cada operación (etapa de comunicación mediante procedimiento documentado).

Por tratarse de un código general, en cada una de estas operaciones se asignan diversas tareas, cuya ejecución puede realizarse en diferentes secuencias de modo parcial o gradualmente, estimando las necesidades de la organización. Es precisamente en el detalle de las tareas señaladas para cada operación en el que se enuncian buena parte de las técnicas de auditoría. La normativa, por tanto, orienta hacia el empleo de determinadas técnicas cuyo conocimiento adquirido presupone. ${ }^{27}$ Seguidamente enumeramos en orden de aparición en la normativa las técnicas explicitadas: interrogación de fuentes documentales, elaboración de entrevistas, análisis del contexto institucional, análisis de debilidades y de fortalezas; análisis de la organización del trabajo: definición y representación de funciones, actividades, operaciones y procesos de negocio (desde la perspectiva documental), identificación de requisitos documentales mediante el análisis del contexto, funcional y secuencial (de procesos), e identificación de estrategias para el diseño. De modo implícito se intuyen la elaboración de bases de datos donde se incorpore la información recopilada, cuestionarios o formularios, el inventario físico de documentos, el análisis de costos y beneficios frente a la formulación de

26 Estas operaciones así como su contenido se desdibujan en el borrador ISO/DIS 30301:2010 en el que se ha primado la regulación de la información que debe ser identificada para la comprensión del contexto de producción en detrimento de la descripción del método y de sus técnicas de investigación.

27 Véanse al respecto los manuales básicos de gestión de documentos anteriormente citados. 
estrategias, la sistematización de datos mediante bases de datos elaboradas; la elaboración de informes; la planificación y ejecución del trabajo auditor; el análisis del riesgo sobre gestión documental; las técnicas de comunicación verbal y escrita, etcétera.

En la tabla siguiente se agrupan dichas técnicas considerando las respectivas fases del método:

Tabla 1- Etapas y técnicas empleadas en la auditoría de requisitos informativos ${ }^{28}$

\begin{tabular}{|c|c|c|}
\hline $\begin{array}{c}\text { Etapas del proceso } \\
\text { auditor }\end{array}$ & Técnicas empleadas & $\begin{array}{c}\text { Operaciones enunciadas en la norma } \\
\text { ISO 15489:2001 }\end{array}$ \\
\hline Recolección de datos & $\begin{array}{l}\text { Observación directa: entrevistas, } \\
\text { formularios específicos } \\
\text { Acompañamiento "in situ" } \\
\text { Inventario físico } \\
\text { Observación indirecta } \\
\text { Explotación de fuentes } \\
\text { Elaboración de cuestionarios }\end{array}$ & Investigación preliminar \\
\hline $\begin{array}{l}\text { Análisis e } \\
\text { interpretación de } \\
\text { datos }\end{array}$ & $\begin{array}{l}\text { Análisis del contexto } \\
\text { Análisis funcional } \\
\text { Análisis secuencial (identificación y } \\
\text { representación de procesos y } \\
\text { actividades) } \\
\text { Elaboración de mapas de procesos } \\
\text { Definición de funciones, procesos y } \\
\text { actividades. } \\
\text { Análisis de riesgos } \\
\text { Análisis de costos cuantitativos } \\
\text { Análisis DAFO }\end{array}$ & $\begin{array}{l}\text { Análisis de actividades (desarrollo } \\
\text { específico de normativa complementaria } \\
\text { ISO 26122: 2008) }\end{array}$ \\
\hline $\begin{array}{l}\text { Identificación de } \\
\text { requisitos y } \\
\text { formulación de } \\
\text { estrategias }\end{array}$ & \begin{tabular}{|l|} 
Tormenta de ideas \\
Identificación y definición de \\
necesidades y expectativas \\
(elaboración de registros de base \\
de datos) \\
Planificación de acciones de mejora \\
Análisis DAFO: estrategias defensivas y \\
ofensivas \\
Gestión de riesgos (estrategias) \\
Análisis de costos (estrategias) \\
Análisis de beneficios (estrategias)
\end{tabular} & $\begin{array}{l}\text { Identificación de requisitos de los } \\
\text { documentos de archivo } \\
\text { Identificación de las estrategias } \\
\text { Evaluación de sistemas activos. }\end{array}$ \\
\hline Comunicación & \begin{tabular}{|l|} 
Elaboración del Informe de auditoría \\
Estrategias de comunicación verbal \\
sustentadas con material visual
\end{tabular} & $\begin{array}{l}\text { Documentación de procesos: } \\
\text { "estrategias documentadas en un plan } \\
\text { estratégico. (aparado 5.3.2 norma) }\end{array}$ \\
\hline
\end{tabular}

28 En el borrador ISO/DIS 30301:2000, la etapa de recolección de datos se desdibuja como tal, y la identificación y los análisis de procesos de trabajo, los riesgos asociados al incumplimiento documental, y los recursos disponibles, así como los requisitos de comunicación del plan de diseño, son trabajados en distintos enunciados de los apartados 6 a 9. 
Destinamos los siguientes subepígrafes de este acápite al análisis de las técnicas que se muestran en la tabla.

\subsection{Técnicas asociadas a la recolección de datos}

La finalidad de la recolección de datos permite conocer los contextos narrativos en los que se imbrican las organizaciones. Se trata de recopilar información sobre el contexto jurídico, administrativo, informativo y social de la institución, su cultura corporativa y de gestión documental así como las expectativas de los productores, clientes y proveedores, con el fin de aproximarse a la institución desde múltiples perspectivas. El intento radica en reunir aquellos datos que faciliten el conocimiento de los contextos en que surgen los documentos para poder sopesar el valor de evidencia de los mismos, su grado de cumplimiento respecto a un marco legislativo, normativo, jurídico y procedimental, e identificar los factores que influyen en las necesidades de crear, disponer y conservar los documentos, con independencia del soporte y sus medios de creación. Permite reconocer fortalezas, debilidades, obstáculos u amenazas, así como aquellas potenciales oportunidades relativas al sistema de gestión de documentos activo en la organización; La recolección de datos contribuye, igualmente, a identificar problemas y/o no conformidades de archivo, y a apoyar la toma de decisiones sobre el sistema de gestión documental. ${ }^{29}$

Dos son los métodos utilizados en la reunión de datos: la observación directa y la indirecta. La observación indirecta se efectúa mediante el uso de técnicas tales como la elaboración de cuestionarios, y la explotación de fuentes internas (políticas, planes, informes sobre estrategias o programas, memorias de actividades, manuales de procedimientos, organigramas, planos, fichas de descripción de puestos, manuales de calidad, mapas de procesos, matrices registro de seguridad, matrices de competencias, inventarios, balances financieros, presupuestos, etc.), y externas a la organización (marco legislativo y normativo glocal, códigos de buenas prácticas, convenios, contratos, especificaciones de clientes, estudios de mercados, etc.) o la valoración de hallazgos derivados de procesos de medición (registros de datos, estallidos de control, aplicación de indicadores, compromisos de servicio, datos sobre rendimientos, herramientas de evaluación, informes de mejora, informes de auditoría, etcétera.)

29 El borrador ISO/DIS 30301(2010: p2) ofrece en el apartado 4 una relación amplia de elementos a investigar sobre el contexto organizacional. 
El método de observación directa se ejerce mediante el manejo de técnicas tales como el empleo de registros y formularios llevados a cabo por personal especializado en el ejercicio de las tareas, la elaboración de entrevistas, la observación directa in situ, o la planificación y elaboración de un inventario físico. La utilidad de las entrevistas y de las técnicas de observación in situ se comprueban en la investigación de contextos operativos complejos, así como en la identificación de las expectativas de los productores. Por otro lado mediante la técnica de inventariado físico de los documentos se establece una radiografía de/(de los) programa(s) de gestión activo(s) en la organización, y se obteniene un conjunto de datos que serán utilizados para el rediseño o la mejora de áreas funcionales del sistema de gestión documental: recursos, infraestructuras, tecnologías, técnicas, procedimientos de archivo, así como valoraciones sobre la cantidad, la calidad y el estado del/los fondos documentales.

\subsection{Técnicas asociadas al análisis e interpretación de datos}

El análisis e interpretación de datos se define como un proceso intelectual de revisión de apuntes coleccionados mediante la aplicación de diversas técnicas. Mientras que la recolección agrupa informaciones y describe situaciones diversas, el análisis permite aunar, confrontar y reflexionar sobre los datos descritos con una lógica sistémica y sistemática basada en interrogantes y alternativas.

En esta línea de confrontación de interrogantes y alternativas, el inventario físico (recolección de datos sobre recursos de gestión documental y archivos) se contrapone al "inventario conceptual" 30 de organización del trabajo (identificación de flujos de información, funciones y procesos), precisamente para poder obtener una panorámica basada en la diferencia percibida entre el funcionamiento de la institución (perspectiva de las necesidades de información) y el tipo de respuesta aportada por el/los programa(s) de gestión documental activo(s); esto es, entre el modelo de requisitos informativos necesitados para potenciar una organización competitiva y el modo de organizar, disponer, utilizar y conservar los documentos. En suma, el modo en que la organización aporta una respuesta a sus necesidades de gestión informativa.

El enfoque de análisis de la organización del trabajo responde a los horizontes novedosos: contribuir de modo benéfico a perfilar y rentabilizar el nivel de gestión de la información requerido por la organización para alcanzar sus

30 El análisis de la organización del trabajo fue denominado por los archiveros australianos "inventario conceptual” para diferenciarlo del físico (centrado en documentos). En sus inicios se trataba de un análisis de los flujos informativos, funcionales y terminológicos. 
metas y, si es posible, contribuir a que se mantenga competitivo en la cresta de la ola de los mercados globales. En correlación con esta técnica de análisis, los datos son interpretados bajo el tamiz del análisis del riesgo. A este respecto cabe señalar que la identificación y la gestión de riesgos, han venido fundamentalmente siendo utilizados en el contexto archivístico para la prevención de desastres, ante posibles paradas del negocio, mediante la identificación y control de documentos esenciales así como para asegurar la conservación de documentos. Esta técnica de análisis se orientaba hacia el objeto documento frente a los emergentes enfoques, donde el riesgo se investiga asociado a la operatividad, imagen, productividad o rendimiento de la organización en general y, de modo específico, asociado al sistema de archivo o al de su arquitectura de los procesos. Por tanto se realiza centrándose en el análisis del trabajo con objeto de comprender los requisitos emergentes y cambiantes de gestión documental y de evaluar su grado de cumplimiento. En el paisaje observado el riesgo delimita incumplimientos actuales y potenciales motivados por factores internos o externos a la organización.

Una tercera técnica de enfoque novedoso en su aplicación hacia el conocimiento de los contextos de la institución, y asociada al conocimiento de los factores internos y externos de la organización, es el análisis DAFO. Mediante dicha técnica se identifican e interpretan fortalezas, debilidades, amenazas y oportunidades, que afectan a la gestión de documentos, con la perspectiva de generar estrategias diversas ofensivas o defensivas y de viabilidad o reorientación.

Finalmente una vez interpretados los datos compilados en el inventario físico de documentos, la información derivada del DAFO y aquélla resultante de la identificación de riesgos, se obtendrán determinados hallazgos a los que se les aplicará una última técnica de análisis: el análisis de los costos que suponen los riesgos identificados, y de los beneficios que subyacen en las estrategias definidas. La investigación de costos y beneficios, tal y como se ha señalado con anterioridad, ha venido siendo utilizada en el ejercicio archivístico desde la perspectiva de rentabilidad económica y desde un enfoque de análisis de activos tangibles; especialmente para la conservación rentable de documentos basada en la gestión de espacios, soportes y para la recuperación de la información, mediante la reducción de tiempos de servicio y de búsqueda. De este modo se distinguían costos y beneficios tangibles. En el anterior enunciado hemos subrayado cómo la transmutación del paisaje organizacional ha permitido ensanchar la perspectiva de los costos y beneficios hacia la consideración estratégica de activos intangibles, cuyo reconocimiento y número es considerable. Entre los costes de esta categoría, destacamos: impacto de no cumplimiento del marco legislativo y reglamentario; impacto del incumplimiento de 
códigos de buenas prácticas que requieren ser certificados; impacto de incumplimiento de códigos de buenas prácticas que inciden en la imagen de la corporación; impacto de incumplimiento de convenios, especificaciones y contratos con los clientes o de retrasos en los mismos; impacto de incumplimiento del código ISO 15489 sobre gestión de documentos; gestión del conocimiento dañado o agravado ante operaciones descentralizadas; reducción de la memoria corporativa motivada por cambios en los procedimientos administrativos o en las responsabilidades así como por cambios continuados o una alta remodelación de la plantilla; costos de litigios potenciales debidos a una inadecuada gestión de documentos y de la información; responsabilidad reducida en la toma de decisiones ante acciones determinadas; descenso de la productividad en la organización, etcétera.

En otro orden, la técnica de análisis de la organización del trabajo editada como informe técnico UNE-ISO/TR 26122:2008, con la perspectiva de mejorar la gestión documental, retoma como planteamiento el siguiente supuesto: en una organización son reconocibles dos planos activos de recolección y análisis de información para (re)diseñar un programa de gestión de documentos: una estructura cartográfica conceptual o lógica y otra física o documental.

La primera se refiere a un plano operativo centrado en la arquitectura corporativa, funcional y secuencial de funciones, procesos, actividades (operaciones) y tareas (transacciones) que permitirán comprender:

a) las relaciones entre diversas sedes de una organización, y los comportamientos y culturas predominantes de gestión de la información "intersede"; 31

b) las relaciones establecidas en cada sede en las que es posible reconocer la arquitectura funcional y la de los procesos $^{32}$;

c) las relaciones establecidas a nivel de función en la cual será posible identificar, describir y representar las relaciones de los procesos y su secuencia de operaciones; ${ }^{33}$

d) listados de riesgos potenciales asociados a los procesos;

e) listas de autoridades derivadas del conjunto de actividades, tareas o transacciones, $\mathrm{y}$

f) listados de requisitos documentales asociados a las actividades, tareas o transacciones.

31 Análisis de la gestión de la información en un entorno corporativo de un organismo con independencia de los locales o los lugares donde se expanda y ubique la organización.

32 Análisis de la gestión de la información en grandes funciones y/o mapa de procesos con base en la categoría de los procesos.

33 Análisis funcional y secuencial. Identificación de funciones y de procesos mediante un mapa de procesos y la definición de éstos. 
Los objetivos de la metodología empleada para la investigación de la organización del trabajo señalados por G Schäfer (1988: p35), se muestran en consonancia con la anterior enumeración y son: obtener una descripción completa de los procesos de negocio; comprender las relaciones de las funciones con el logro de metas y objetivos; actuar cómo guía para facilitar soluciones técnicas alternativas, y ofrecer pautas para la valoración de posibles soluciones y para la selección de instrumentos y técnicas de gestión.

La segunda estructura del informe técnico ISO/TR 26122 consiste en un plano documental o tangible; se trata de normalizar el desarrollo de una cartografía investigada a partir de los modos de organizar los documentos considerados, estos últimos como evidencias directas de la información requerida, surgida en los procesos del negocio, y en las que es posible diferenciar:

a) datos y documentos exógenos y endógenos en los procesos y agrupaciones documentales (reales y potenciales: conformados a partir de posibles combinaciones de datos);

b) depósitos de datos y de documentos, y

c) directorios de información sobre lo almacenado en los depósitos y/o repositorios de datos y de documentos: instrumentos de referencia, etcétera.

Las discrepancias observadas entre los resultados obtenidos del análisis de la organización del trabajo, del análisis de riesgos asociados y de los costos, respecto a los hallazgos del inventario físico y del DAFO, facilitan la delimitación de prototipos funcionales del sistema de gestión de documentos (reales y necesarios): modelo funcional y del flujo de información; prototipo de clasificación; modo de denominación, tesaural o de control de autoridades; tipo de acceso/restricción a la información; modelo de control y gestión de la información electrónica; (esquema(s) de metadatos, modelo de digitalización, etc.); prototipo de valoración y disposición de documentos; arquetipo de riesgos asociados a áreas y subáreas sobre gestión de documentos; prototipo de competencias requeridas para la gestión de documentos, prototipo de servicio de información, etcétera.

A nuestro entender la importancia adquirida en el diseño eficiente de sistemas de gestión de documentos, de estas técnicas de análisis de la organización del trabajo, de identificación y administración de riesgos y de investigación de los costes y beneficios, anuncian novedosas perspectivas y enfoques para el ejercicio profesional: en el modo de "Archivalizar”. ¡O viceversa! Los incipientes contextos reflejados en el cambio constante de requisitos de información, encaminan al archivero hacia el manejo de estas técnicas. 


\subsection{Técnicas asociadas a la identificación de requisitos y formulación de estrategias}

La tercera etapa de auditoría: identificación de requisitos y formulación de estrategias, tiene como finalidad:

1) identificar cada requisito de gestión documental de tal modo que permita alimentar y mantener una base de datos activa de requisitos de gestión, y

2) definir estrategias de gestión documental sobre: a) regulación del flujo documental (documentos activos, semiactivos e inactivos; b) determinación de documentos de archivo o no archivo y su gestión: eliminación, conservación, destrucción y gestión de duplicados; c) concurrencia y gestión de documentos electrónicos d) tecnologías; e) seguridad y protección de los documentos; f) sistemas de clasificación, ordenación, valoración, acceso, indización, descripción y representación; g) sistemas específicos de protección, vitales, de calidad, medioambientales y de seguridad; h) infraestructuras: espacios, depósitos, y equipamiento; i) personal involucrado, competencias y habilidades; j) modalidades de servicios; k) procedimientos y modos de normalizar el trabajo de gestión. Estrategias, todas ellas, orientadas a mantener una gestión de documentos sostenible.

Para su concreción existen variadas técnicas entre las que destacamos las siguientes: la tormenta de ideas que facilita la enumeración de requisitos y la búsqueda de estrategias; la identificación y definición de necesidades y expectativas mediante registros o formularios creados a tal fin; las técnicas de planificación de acciones de mejora que permiten su identificación y priorización; la definición de estrategias defensivas, ofensivas, de viabilidad y de reorientación derivadas del análisis DAFO; la gestión del riesgo, una vez identificado mediante el análisis, y finalmente, el análisis de costos de cada estrategia así como de los beneficios derivados de su propuesta.

\subsection{Técnicas asociadas a la comunicación}

Esta etapa final de auditoría que hemos denominado comunicación, tiene por finalidad informar sobre los hallazgos de auditoría y proponer estrategias definidas en función de dichos hallazgos, para contribuir a una toma de decisiones sobre el diseño o mejora del sistema de gestión documental. Aunque el principal medio de comunicación es el lenguaje verbal directo, establecido entre 
dos o más personas, en las instituciones, éste no siempre es factible y es el medio escrito el predominante. Himstreet y Baty (p.141) señalan que para la elaboración de un informe es preciso considerar los siguientes apartados: determinación del problema, descripción del método utilizado y relación de las fuentes consultadas, información sobre los resultados encontrados, conclusiones y (en su caso) recomendaciones (sobre los resultados y en relación al problema abordado). De este modo, se utiliza "el informe de resultados", entendido como tipología documental que sistematiza de modo coordinado el proceso auditor, los hallazgos y las estrategias. Esto es, informa sobre:

a) cómo se ha llevado a cabo el proceso auditor: métodos, técnicas, equipo e incidencias;

b) los principales resultados obtenidos: actividad real, entorno interno y externo, compromisos de gestión, principales apoyos, riesgos, costos de incumplimiento; $y$

c) la enumeración de estrategias de diseño o de mejora de la situación o el aspecto analizado del sistema: beneficios, opciones de reorientación, recursos necesarios y mecanismos de control y de seguimiento.

En otro orden, la complejidad del proceso, debido al número de técnicas que es factible coordinar, y el impacto añadido que conlleva el re(diseño) de un sistema de gestión de documentos en una organización, exigen una adecuada planificación. El proyecto auditor debe incluir, al menos, los objetivos formulados de auditoría, la selección de instrumentos más adecuados para su ejecución, los recursos materiales y humanos así como la infraestructura tecnológica necesaria, las competencias y habilidades requeridas para el desempeño auditor, las responsabilidades asignadas en la ejecución, un cronograma del proceso auditor, y un presupuesto realista y desglosado. ${ }^{34}$

34 El borrador ISO/DIS 30301 (2010: p5) incluye un apartado específico sobre la implementación del plan de gestión documental que debe ser documentado. En él se informa sobre: "identificación y provisión de recursos adecuados incluyendo aquellos tecnológicos y presupuestarios para el logro de los objetivos; asignación de los recursos al personal responsable para el logro de los objetivos, definición y asignación de responsabilidades para lograr los objetivos documentales propios de los procesos de trabajo; definir estrategias y programas mediante los cuáles se hará efectiva la gestión documental". 


\section{EXPERIENCIAS INTERNACIONALES Y NACIONALES: HACIA LA BÚSQUEDA DE SIGNIFICADOS}

"Me parece que yo veo algún significado...Y yo a nadar no aprendí.. Tú no sabes nadar ¿o sí? ${ }^{35}$ En el País de las maravillas a muchos de sus súbditos les resultaba imposible nadar, dada su naturaleza y constitución: parte de ellos eran una baraja de cartón. Ulises, un estupendo marino, requirió tiempo y experiencia para divisar Ítaca. Por el contrario, en la comunidad archivística, resulta notable el número de archiveros que nadan en las aguas de la auditoría de requisitos informativos y documentales, y de cuya experiencia es posible aprender.

En el plano internacional, resulta obligado citar a los Archivos Nacionales de Australia y de Nueva Zelanda, los cuales han dispuesto instrumentos de formación (manual ${ }^{\text {DIRKS})}$ e información (análisis del trabajo, instrumentos básicos de gestión, etc.) sobre su trabajo, ${ }^{36}$ cuyo resultado es una buena muestra sobre la utilización de la auditoría. De la observación de sus herramientas de trabajo, destacamos que en aquéllas destinadas al análisis del trabajo se han incorporado formularios específicos de análisis, en los que se ha estimado el riesgo así como la incorporación de posibles requisitos documentales.

De igual modo resultó muy interesante la propuesta descriptiva del trabajo de los Archivos de educación superior de Escocia (GASHE), cuyo resultado se debe a un proyecto de investigación de los Servicios de Archivos Universitarios de Glasgow, acometido entre el 2003 y el 2006. ${ }^{37}$ En dicha propuesta, converge una nueva tendencia de descripción acerca de las funciones y actividades cuya formalización editará el Consejo Internacional de Archivo bajo las siglas ISDF: la descripción del trabajo. ${ }^{38}$ Análisis del trabajo y descripción del mismo confluyen aunque, con idéntico proceder que en el País de las maravillas, el único modo de secar dos normas que nadan en el mismo charco es mediante una carrera en equipo. ${ }^{39}$ En este sentido, urge un análisis de los elementos concurrentes de dichas normas, además de una reflexión sobre la

35 Opus cit. p.199.

36 La referencia a los Archivos Nacionales de Australia se incluye en la nota 14. La visualización en línea de estos instrumentos es factible para Nueva Zelanda en el sitio que seguidamente se especifica: [http://continuum.archives.govt.nz/recordkeeping-publications. html\#standards], (Fecha de consulta: 21-10-2010).

37 Arts and Humanities Research Council: Developing Archival Context Standards for Functions in the Higher Education Sector. Final Report, [http://www.gashe.ac.uk/about/standard.html] (data de consulta: 10-10-2010).

38 Consejo Internacional de Archivos: ISDF. Norma internacional para la descripción de funciones, $1^{\mathrm{a}}$ ed. Dresde; 2007.

39 Retomando la propuesta que en el País de las Maravillas realiza ese extraño animal denominado Dodo, p. 43. 
necesidad de colaboración conjunta de los distintos organismos ante objetivos similares y/o colindantes. Sin lugar a dudas los aspectos descriptivos de contenido se recolectan, de igual modo, al hacer el análisis de la organización del trabajo. La elaboración de la norma ISDF testimonia la importancia de la investigación del trabajo en las organizaciones.

En la esfera española la publicación de los procedimientos administrativos de la Universidad Pública de Navarra (2003), es fiel reflejo de la importancia dada a la organización del trabajo, y contribuye a la transparencia de la entidad y a la comunicación "entre la racionalidad de la práctica administrativa y los usuarios" de la institución. Se trata de un trabajo elaborado por una Comisión constituida por profesionales provenientes de diferentes sectores: ${ }^{40}$ esta colaboración expresa cómo dicho análisis y descripción se circunscriben a la organización, y avala la postura de "exterioridad del archivo" defendida en este estudio. ${ }^{41}$ A este tenor opinamos que la investigación del trabajo en una organización debe afrontarse en colaboración con otros sectores de la organización ante los entornos de trabajo electrónico, donde la comprensión y sistematización de éste es requisito imprescindible para hacer factible cualquiera de los servicios electrónicos dirigidos a los ciudadanos.

El e-gobierno en España se manifiesta, desde la perspectiva de gestión documental, en la experiencia del principado de Asturias; comunidad que ofrece un servicio a su ciudadanía autonómica en un entorno de servicio electrónico. Los avances en este campo permitirán incluir elementos de análisis de riesgos y de requisitos documentales, facilitando, en estos entornos, estrategias de gestión de metadatos mediante una infoestructura cercana a la administración y al administrado (Sánchez Vicente; 2009).

El análisis del trabajo en el ejercicio de archivalizar se expande hasta el diseño del sistema de archivos y su arquitectura de procesos. En la actualidad, son numerosos los centros de archivo que tienen experiencia en el análisis y descripción de las funciones y procesos específicos a sus sistemas de archivo. ${ }^{42}$ En esta línea, a modo de ejemplo, se referencia a la Comunidad de

40 La Comisión está formada por juristas, profesores de Derecho e Historia, directores de servicio y técnicos del archivo.

41 En este caso, la descripción y representación gráfica de los procedimientos administrativos incluye algunos elementos que hacen referencia al archivo: código clasificatorio, expediente de la Comisión de Archivo, página Web, documentos asociados, etcétera.

42 El borrador de la norma ISO/DIS 30301 (2010: p9) señala los siguientes procesos en función de la creación de documentos y de su control: procesos de creación: concreción y captura de documentos en cada proceso; gestión de metadatos antes de su incorporación al sistema, gestión de soporte, formato y estructura, y gestión de tecnología para la creación y captura de datos en el sistema. Procesos de control: gestión de metadatos durante y después de su incorporación, gestión de su acceso y restricción de la información, gestión de su disponibilidad, gestión de su valoración y selección, gestión de condiciones para el mantenimiento y administración de sistemas de archivos. 
Castilla y León quien en su III Plan de Intervención del Patrimonio documental (2010:p 16375 y ss.) establece, en su estrategia de Gestión integral de archivos, programas específicos de organización y gestión y de normalización orientados a la "consecución de una Administración más eficaz y eficiente", "potenciando la implantación de la gestión por procesos en todos los centros y órganos del sistema".

Finalmente señalamos una última y sobresaliente experiencia de identificación y representación del trabajo mediante mapa de procesos, así como del trabajo de archivo. Se trata de la realizada por la Unidad de Documentación, Archivo y Registro de la Universidad Miguel Hernández. ${ }^{43}$

\section{Conclusiones}

El profesional del futuro debe afrontar un liderazgo en materia de gestión documental que sea capaz de

comunicar a la organización la importancia de asegurar el cumplimiento de los requisitos de gestión documental; de establecer, comunicar y revisar políticas y objetivos de gestión documental; de implantar revisiones y mecanismos de control, y de asegurar la disponibilidad de los recursos (ISO/DIS:2010:p3)

para hacer factible un sistema de archivo sostenible; esto es, estratégico para la organización. En este estudio hemos demostrado cómo el profesional se ha orientado hacia el cambio e intenta adaptarse él, mediante: a) una reflexión sobre los nuevos contextos de producción en los que ejerce su trabajo; b) una revisión de arquetipos y c) la incorporación de un nuevo método de gestión, basado en el ciclo PDCA y similar a los adoptados por otros sistemas de gestión: calidad, seguridad, etc. El avance es significativo ya que de una posición de ejercicio profesional basada en la especificidad se ha derivado hacia una normalización del trabajo mediante la concreción de un método de aplicación universal.

En este trabajo han sido revisados múltiples factores que inciden en el desarrollo de modelos de información y gestión documental en las organizaciones. Factores heterogéneos relacionados con niveles informativos, dimensiones de información, valores culturales y éticos, pervivencia de sistemas de gestión, entornos tecnológicos y de comunicación complejos, competitividad

43 Esta autora agradece a $\mathrm{M}^{\mathrm{a}}$ José Martínez Gómez su gentileza y presteza para responder a la información demandada sobre los procesos vigentes en la Unidad de Documentación, Archivo y Registro de la UMH. 
de mercado, etc., aunque homogéneos en su finalidad: mantener sistemas de producción organizacional competitivos para cuyo alcance se requiere un arquetipo de gestión documental eficiente y colaborativo. El resultado transmuta la imagen que las organizaciones tenían de su(s) sistema(s) de gestión documental, y de un modelo pasivo de recepción de documentos y/o activo en lo referente a la consecución de objetivos departamentales se ha derivado a una visión estratégica del arquetipo, más cercana a un activo intangible que permite y asegura la continuidad de negocios, el cumplimiento de requisitos documentales derivados de un marco legislativo y normativo cada vez más complejo, una visión corporativa y de entornos de mercado más amplia que de este modo responde a las expectativas y necesidades de usuarios, shareholders (accionistas) y (los depositarios de las apuestas) stakeholders.

Desde esta perspectiva de cambio, la planificación de la gestión de documentos resulta vital e imprescindible, pues sólo a través de ella es posible conocer contextos de producción y requisitos de gestión documental. Hemos afrontado en este estudio los métodos de gestión de un sistema de archivos basado en el ciclo PDCA, centrándonos en la fase de planificación y más concretamente en la auditoría de requisitos de gestión documental. Hemos revisado los métodos y técnicas empleados desde una perspectiva retrospectiva y prospectiva, y apuntado enfoques emergentes en su utilización e interpretación. Es precisamente esta visión sobre la comprensión de los contextos de producción en las organizaciones la que actualmente tiende a primarse. Aunque, tal y como hemos subrayado en esta investigación, la auditoría de requisitos informativos aporta, además, otro tipo de conocimientos indispensables o complementarios para el diseño, de implementación y la medición de sistemas de gestión documental, pues nos permite conocer los contextos externos a la organización y sus expectativas.

Hemos intentando demostrar cómo una administración eficaz y eficiente requiere líneas estratégicas de actuación sobre sus niveles de información. El conocimiento de la necesidades de información y los requisitos de gestión documental asociados a la organización del trabajo, facilitarán el logro de actuaciones administrativas eficaces, y asegurarán servicio, operatividad y cumplimiento. El archivero ha sabido crear una normativa para su análisis (UNE-EN-ISO 26122:2008) y otra para su descripción (ISDF). A este respecto, hemos apuntado que el método y el instrumento deben ser coordinados y difundidos de modo conjunto para evitar solapamientos y facilitar el trabajo de ambos procesos: el de análisis y el de descripción.

La identificación —y gestión — de los riesgos asociados al no cumplimiento de requisitos documentales, la identificación de las inconformidades, el análisis detallado de costos y beneficios, el conocimiento de las expectativas 
de los usuarios e interesados específicos para cada categoría de los procesos del negocio, contribuirán a lograr una administración eficiente, además de preservar el patrimonio documental corporativo. El método para desarrollar el análisis y la gestión de riesgos debe ser completado a la luz de la normativa existente relacionada con la de gestión de riesgos. A tal efecto es posible enriquecer el trabajo mediante la consulta de normas específicas para sistemas de gestión, tales como la norma ISO 31300:2009.

Por otro lado la operatividad de bases de datos específicas sobre requisitos de información y las estrategias de implementación, facilitan la orientación de una administración hacia la mejora continuada, a la par que establecen los mecanismos para orientar el sistema de gestión documental hacia su mejoramiento. Enfoque este último que adquiere gran relevancia en el borrador de la normativa ISO/DIS 30301:2010, donde certificación y mejoramiento se contemplan ampliamente desarrollados. En este sentido la normativa DIS incluye una futura norma sobre evaluación (código: 30304), y otra sobre certificación (código: 30303); su elaboración, edición e implantación permite augurarle a la gestión documental un importante futuro.

En definitiva, la auditoría de requisitos fundamenta la lógica de los objetivos de logro formulados en el diseño de un sistema de gestión documental, y por lo tanto se torna imprescindible para medir el rendimiento y los resultados esperados. Su relevancia es tal que asegura un Archivo sostenible y derriba las altas y sólidas murallas levantadas en su derredor, "porque [el profesional] muchas cosas tenía que hacer fuera", haciendo referencia a otro magnífico poema de C. Cavafis. ${ }^{44}$

\section{ReCursos Bibliografícos}

Aenor (2005): UNE-EN-ISO 9000:2005, Sistemas de gestión de calidad. Fundamentos y vocabulario, Madrid, AENOR (2008): UNE-EN-ISO 9001:2008, Sistemas de gestión de calidad, Requisitos, Madrid, AENOR. (2000), UNE-EN-ISO 9004:2000, Sistemas de gestión de calidad. Directrices para la mejora del desempeño, Madrid, AENOR. Esta norma ha sido revisada. Véase, UNE-EN-ISO 9004:2009, Gestión para el éxito sostenido de una organización. Enfoque de gestión de la calidad, Madrid.

44 Opus. cit. Poema "Murallas": Sin miramiento, sin pudor, sin lástima/ altas y sólidas murallas me han levantado en torno./ Y ahora, heme aquí, quieto y desesperándome,/ No pienso en otra cosa: este destino me devora el alma;/ porque yo muchas cosas tenía que hacer fuera. ;Ay, cuando levantaban las murallas, cómo no me di cuenta! Pero nunca oí ruido ni voces de albañiles./ Imperceptiblemente me encerraron fuera del mundo, p. 50. 
, (2002): UNE-EN-ISO 19011:2002, Directrices para la auditoría de los sistemas de gestión de la calidad y/o ambiental, Madrid: AENOR.

, (2002) UNE-66925: 2002, Directrices para la documentación de sistemas de gestión de la calidad, Madrid, AENOR (ISO 10013:2001). , (2006): UNE ISO 15489:2006, Información y Documentación. Gestión de documentos de archivo. Parte 1, General, Madrid: AENOR, (ISO 15489:2001).

(2006): UNE ISO 15489:2006, Información y Documentación. Gestión de documentos de archivo. Parte 2, Directrices, Madrid. AENOR, (ISO/TR 15489-2:2001).

, (2008): UNE ISO TR 26122 2008, Análisis de procesos de trabajo para [la gestión de] documentos. Madrid: AENOR (ISO TR 26122:2007).

(2009): UNE-EN-ISO 31000:2009, Gestión del riesgo. Principios y directrices, Madrid: AENOR.

D’Alós-Moner, A. (2001), "El profesional del siglo XXI al servicio de la sociedad y de las organizaciones"., en El profesional de la Información, v. 10, núm.12, pp 26-29; Bustelo Ruesta, C. (2003), "Gestión documental y gestión de contenidos en las empresas: estado del arte 2002 y perspectiva para el 2003”, en El profesional de la Información, v. 12, núm. 2, pp. 118-120.

Casellas i Serra, L. E. (2009), "La profesión en tiempos de cambio", Tábula, núm. 12, pp. 33-46.

Davis, J. Millar, G. y Russell, A. (2008), La Revolución de la Información. Cómo utilizar el modelo de evolución de la información para que su empresa crezca, Barcelona: Bresca Profit.

Heredia Herrera, A. (2007), ¿Qué es un archivo?, Gijón: Trea.

Hinstreet, Willian C. y Batyt Wyne M (2000), Guía para la redacción de carta e informes en la empresa, Bilbao: Ediciones Deusto.

ISO (2010): ISO/DIS 30300:2010, Management system for records- Fundamentals and vocabulary Ginebra: ISO.

ISO (2010): ISO/DIS 30301:2010, Management system for records- Requirements- Ginebra: ISO.

Junta de Castilla y León (2010), Acuerdo 18/2010 del 18 de Febrero de la Junta de Castilla y León, por el que se aprueba el III Plan de Intervención en el Patrimonio Documental de Castilla y León, 2010-2015 (BOCYL Núm. 37, 24/02/2010).

Katelaar, E. (2001),"Tacit Narratives: the Meanigns of Archives”, en Archival Science, v. 1, núm. 2, pp 131-141, (traducido al español en Tábula 10;2007).

Llansó SanJuán, J. (2009), "Camino a Ítaca. El papel de la normalización en el periplo profesional del archivero para la construcción de su identidad", en Tábula, núm.10, pp. 205-234. 
Sánchez Vicente, P. (2009), "La gestión de documentos electrónicos en la E-Administración”, en I Seminario Internacional. Archivos y documentos electrónicos, Bogotá: 11-13 Marzo, 2009, editado en Actas CD-Rom, Universidad de La Salle.

Skupsky, Donald S. (1994), Recordkeeping requirements. The First Practical Guide to Help You Control. Your Records...What You Need to Keep and What You Can Safely Destroy, Denver: Information Requirements Clearinghouse, $4^{\mathrm{a}}$ ed.

Schafer, G. et al. (1988), Functional analysis of office requirements: a multiperspective approach, Chichester: John Wiley \& Sons, 1988.

Universidad Pública de Navarra (2003), Manual de procedimientos administrativos, Navarra: Universidad. 